\title{
THE ARGUMENT SHIFT METHOD AND MAXIMAL COMMUTATIVE SUBALGEBRAS OF POISSON ALGEBRAS
}

\author{
Dmitri I. Panyushev and Oksana S. Yakimova
}

\section{Introduction}

Let $\mathfrak{q}$ be a Lie algebra over an algebraically closed field $\mathbb{k}$ of characteristic zero. The symmetric algebra $\mathcal{S}(\mathfrak{q})$ has a natural structure of Poisson algebra, and our goal is to present a sufficient condition for the maximality of Poisson-commutative subalgebras of $\mathcal{S}(\mathfrak{q})$ obtained by the argument shift method. Study of Poisson-commuttive subalgebras of $\mathcal{S}(\mathfrak{q})$ has attracted much attention in the last years, see $[2,6,14,15,16]$. This is related to commutative subalgebras of the enveloping algebra $\mathcal{U}(\mathfrak{q})$, fine questions of symplectic geometry, and integrable Hamiltonian systems. Commutative subalgebras of $\mathcal{U}(\mathfrak{q})$ (e.g., the famous Gelfand-Zetlin subalgebra of $\mathcal{U}\left(\mathfrak{s} \mathfrak{l}_{n}\right)$ ) occur in the theory of quantum integrable systems and have interesting application in representation theory.

Let $\mathcal{Z}(\mathfrak{q})$ be the centre of the Poisson algebra $\mathcal{S}(\mathfrak{q})$. For $\xi \in \mathfrak{q}^{*}$, let $\mathcal{F}_{\xi}(\mathcal{Z}(\mathfrak{q}))$ denote the algebra generated by the $\xi$-shifts of all $f \in \mathcal{Z}(\mathfrak{q})$ (see Subsection 2.2 for precise definitions). As is well-known, $\mathcal{F}_{\xi}(\mathcal{Z}(\mathfrak{q}))$ is a Poisson-commutative subalgebra of $\mathcal{S}(\mathfrak{q})$. Furthermore, $\operatorname{trdeg}\left(\mathcal{F}_{\xi}(\mathcal{Z}(\mathfrak{q}))\right) \leqslant(\operatorname{dim} \mathfrak{q}+$ ind $\mathfrak{q}) / 2=: b(\mathfrak{q})$. We say that $\mathcal{F}_{\xi}(\mathcal{Z}(\mathfrak{q}))$ is of maximal dimension, if the equality holds. However, even in this case, it may happen that there is a strictly larger Poisson-commutative subalgebra (of the same transcendence degree). We say that $\mathcal{F}_{\xi}(\mathcal{Z}(\mathfrak{q}))$ is maximal, if it is maximal with respect to inclusion among the commutative subalgebras of $\mathcal{S}(\mathfrak{q})$. Let $\mathfrak{q}_{\text {reg }}^{*}$ denote the set of regular elements of $\mathfrak{q}^{*}$, i.e., those whose stabiliser in $\mathfrak{q}$ has the minimal dimension. For the purposes of this introduction, we state our main result (Theorem 3.2) in a slightly abbreviated form:

Theorem 0.1. Suppose that

(i) $Z(\mathfrak{q})$ contains algebraically independent homogeneous polynomials $f_{1}, \ldots, f_{l}$, where $l=\operatorname{ind} \mathfrak{q}$, such that $\sum_{i=1}^{l} \operatorname{deg} f_{i}=b(\mathfrak{q})$;

(ii) $\operatorname{codim}\left(\mathfrak{q}^{*} \backslash \mathfrak{q}_{r e g}^{*}\right) \geqslant 3$.

Then, for any $\xi \in \mathfrak{q}_{\text {reg }}^{*}, \mathcal{F}_{\xi}(\mathcal{Z}(\mathfrak{q}))$ is a polynomial algebra of Krull dimension $b(\mathfrak{q})$ and it is a maximal Poisson-commutative subalgebra of $\mathcal{S}(\mathfrak{q})$.

Obviously, Theorem 0.1 applies if $\mathfrak{q}$ is semisimple, and we thus generalise results of A. Tarasov [16]. (He proved maximality if $\xi$ is regular semisimple.) There are also other interesting classes of Lie algebras satisfying the conditions of this theorem, see Section 4 .

Received by the editors February 20, 2007.

The first author is partially supported by R.F.B.R. grants $05-01-00988$ and 06-01-72550. The second author is supported by the Alexander von Humboldt-Stiftung. 
A general criterion of Bolsinov [1] asserts that, for $\xi \in \mathfrak{q}_{\text {reg }}^{*}, \mathcal{F}_{\xi}(\mathcal{Z}(\mathfrak{q}))$ is of maximal dimension if and only if codim $\left(\mathfrak{q}^{*} \backslash \mathfrak{q}_{r e g}^{*}\right) \geqslant 2$. For the proof of Theorem 0.1, we need, however, a stronger result. Namely, we provide a precise description of pairs $\xi, \eta \in \mathfrak{q}^{*}$ such that the differentials at $\eta$ of all functions from $\mathcal{F}_{\xi}(\mathcal{Z}(\mathfrak{q}))$ generate a subspace of dimension $b(\mathfrak{q})$, see Theorem 2.5 .

Notation. If an algebraic group $Q$ acts on an irreducible affine variety $X$, then $\mathbb{k}[X]^{Q}$ is the algebra of $Q$-invariant regular functions on $X$ and $\mathbb{k}(X)^{Q}$ is the field of $Q$ invariant rational functions. If $\mathbb{k}[X]^{Q}$ is finitely generated, then $X / / Q:=\operatorname{Spec} \mathbb{k}[X]^{Q}$, and the quotient morphism $\pi_{X}: X \rightarrow X / / Q$ is the mapping associated with the embedding $\mathbb{k}[X]^{Q} \hookrightarrow \mathbb{k}[X]$.

If $V$ is a $Q$-module and $v \in V$, then $\mathfrak{q}_{v}$ is the stabiliser of $v$ in $\mathfrak{q}$. For the adjoint representation of $\mathfrak{q}$, the stabiliser of $x \in \mathfrak{q}$ is also denoted by $\mathfrak{z}_{\mathfrak{q}}(x)$, and we say that $\mathfrak{z}_{\mathfrak{q}}(x)$ is the centraliser of $x$.

All topological terms refer to the Zariski topology. If $M$ is a subset of a vector space, then $\operatorname{span}(M)$ denotes the linear span of $M ; \mathbb{k}^{\times}:=\mathbb{k} \backslash\{0\}$.

\section{On the codim $-n$ property for the coadjoint representation}

Let $Q$ be a connected algebraic group with Lie algebra $\mathfrak{q}$. We write $\mathcal{S}(\mathfrak{q})$ for the symmetric algebra of $\mathfrak{q}$. Recall that $\mathcal{S}(\mathfrak{q}) \simeq \mathbb{k}\left[\mathfrak{q}^{*}\right]$ is a Poisson algebra, and the symplectic leaves in $\mathfrak{q}^{*}$ are precisely the coadjoint orbits of $Q$. Since each coadjoint orbit $Q \cdot \xi$ is a symplectic variety, $\operatorname{dim} Q \cdot \xi$ is even. Let $\{$,$\} denote the Lie-Poisson$ bracket in $\mathcal{S}(\mathfrak{q})$. Then the algebra of invariants $\mathbb{k}\left[\mathfrak{q}^{*}\right]^{Q}=\mathcal{S}(\mathfrak{q})^{Q}$ is the centre of $(\mathcal{S}(\mathfrak{q}),\{\}$,$) . We also write \mathcal{Z}(\mathfrak{q})$ for this centre.

Let $\mathfrak{q}_{\text {reg }}^{*}$ denote the set of all $Q$-regular elements of $\mathfrak{q}^{*}$. That is,

$$
\mathfrak{q}_{\text {reg }}^{*}=\left\{\xi \in \mathfrak{q}^{*} \mid \operatorname{dim} Q \cdot \xi \geqslant \operatorname{dim} Q \cdot \eta \text { for all } \eta \in \mathfrak{q}^{*}\right\} .
$$

As is well-known, $\mathfrak{q}_{r e g}^{*}$ is a dense open subset of $\mathfrak{q}^{*}$.

Definition 1. We say that the coadjoint representation of $\mathfrak{q}$ has the codim $-n$ property if $\operatorname{codim}\left(\mathfrak{q}^{*} \backslash \mathfrak{q}_{r e g}^{*}\right) \geqslant n$.

If $\xi \in \mathfrak{q}_{r e g}^{*}$, then $\operatorname{dim} \mathfrak{q}_{\xi}$ is called the index of $\mathfrak{q}$, denoted ind $\mathfrak{q}$. By Rosenlicht's theorem, trdeg $\mathbb{k}\left(\mathfrak{q}^{*}\right)^{Q}=$ ind $\mathfrak{q}$. It follows that if $f_{1}, \ldots, f_{r} \in \mathbb{k}\left[\mathfrak{q}^{*}\right]^{Q}$ are algebraically independent, then $r \leqslant \operatorname{ind} \mathfrak{q}$. Set $b(\mathfrak{q})=(\operatorname{dim} \mathfrak{q}+\operatorname{ind} \mathfrak{q}) / 2$. If $\mathfrak{q}$ is semisimple, then $b(\mathfrak{q})$ is the dimension of a Borel subagebra.

Example. If $\mathfrak{g}$ is reductive, then ad $\simeq \operatorname{ad}^{*}$ and $\operatorname{codim}\left(\mathfrak{g} \backslash \mathfrak{g}_{\text {reg }}\right) \geqslant 3$. Hence the coadjoint representation of a reductive Lie algebra has the codim-3 property.

The following example pointed out by E.B. Vinberg shows that for any $n$ there are noncommutative Lie algebras with codim $-n$ property.

Example 1.1. Suppose $s \in \mathfrak{g l}(V)$ is a semisimple linear transformation with nonzero rational eigenvalues. Let $\mathfrak{q}$ be the semi-direct product of the 1-dimensional toral Lie algebra $\mathbb{k} s$ and $V$. The Lie bracket is given by

$$
\left[(\alpha s, v),\left(\beta s, v^{\prime}\right)\right]=\left(0, \alpha s\left(v^{\prime}\right)-\beta s(v)\right), \quad \alpha, \beta \in \mathbb{k} .
$$

It is easily seen that ind $\mathfrak{q}=\operatorname{dim} \mathfrak{q}-2$. Moreover, let $L$ be the annihilator of $V$ in $\mathfrak{q}^{*}$. Then the line $L$ is precisely the set of $Q$-fixed points in $\mathfrak{q}^{*}$, while $\operatorname{dim} Q \cdot \xi=2$ for any $\xi \in \mathfrak{q}^{*} \backslash L$. Thus, $\mathfrak{q}$ has the codim $-n$ property with $n=\operatorname{dim} V$. 
If $f \in \mathcal{S}(\mathfrak{q})$, then the differential of $f, d f$, can be regarded as a polynomial mapping from $\mathfrak{q}^{*}$ to $\mathfrak{q}$, i.e., an element of $\operatorname{Mor}_{Q}\left(\mathfrak{q}^{*}, \mathfrak{q}\right) \simeq \mathcal{S}(\mathfrak{q}) \otimes \mathfrak{q}$. More precisely, if $f \in \mathcal{S}^{d}(\mathfrak{q})$, then $d f$ is a polynomial mapping of degree $d-1$, i.e., an element of $\mathcal{S}^{d-1}(\mathfrak{q}) \otimes \mathfrak{q}$. We write $(d f)_{\xi}$ for the value of $d f$ at $\xi \in \mathfrak{q}^{*}$. Recall that $(d f)_{\xi}$ is an element of $\mathfrak{q}$ that is defined as follows. If $\nu \in \mathfrak{q}^{*}$ and $\langle$,$\rangle denotes the natural pairing between \mathfrak{q}$ and $\mathfrak{q}^{*}$, then

$$
\left\langle(d f)_{\xi}, \nu\right\rangle:=\text { the coefficient of } t \text { in the Taylor expansion of } f(\xi+t \nu) .
$$

The rôle of the codim-2 property is seen in the following result, see [11, Theorem 1.2].

Theorem 1.2. Suppose that $\left(\mathfrak{q}, \mathrm{ad}^{*}\right)$ has the codim-2 property and $\operatorname{trdeg} \mathbb{k}\left[\mathfrak{q}^{*}\right]^{Q}=$ ind $\mathfrak{q}$. Set $l=$ ind $\mathfrak{q}$. Let $f_{1}, \ldots, f_{l} \in \mathbb{k}\left[\mathfrak{q}^{*}\right]^{Q}$ be arbitrary homogeneous algebraically independent polynomials. Then

(i) $\sum_{i=1}^{l} \operatorname{deg} f_{i} \geqslant b(\mathfrak{q})$;

(ii) If $\sum_{i=1}^{l} \operatorname{deg} f_{i}=b(\mathfrak{q})$, then $\mathbb{k}\left[\mathfrak{q}^{*}\right]^{Q}$ is freely generated by $f_{1}, \ldots, f_{l}$ and $\xi \in$ $\mathfrak{q}_{\text {reg }}^{*}$ if and only if $\left(d f_{1}\right)_{\xi}, \ldots,\left(d f_{l}\right)_{\xi}$ are linearly independent.

The second assertion in (ii) can be regarded as a generalisation of Kostant's result for reductive Lie algebras [4, (4.8.2)]. Its geometric meaning is the following. Consider the quotient morphism $\pi: \mathfrak{q}^{*} \rightarrow \mathfrak{q}^{*} / / Q \simeq \mathbb{A}^{\text {ind } \mathfrak{q}}$. Then $\pi$ is smooth at $\xi \in \mathfrak{q}^{*}$ if and only if $\xi \in \mathfrak{q}_{r e g}^{*}$.

\section{The argument shift method and Bolsinov's criterion}

2.1. Commutative subalgebras of $\mathcal{S}(\mathfrak{q})$. Let $\mathcal{A}$ be a subalgebra of the symmetric algebra $\mathcal{S}(\mathfrak{q})$. Then $\mathcal{A}$ is said to be Poisson-commutative if the restriction of $\{$, to $\mathcal{A}$ is zero. Abusing the language, we will usually omit "Poisson" and merely say that $\mathcal{A}$ is commutative. Notice that the words "subalgebra of $\mathcal{S}(\mathfrak{q})$ " always refer to the usual (associative and commutative) structure of the symmetric algebra, while "commutative" refers to the Poisson structure on $\mathcal{S}(\mathfrak{q})$.

For any subalgebra $\mathcal{A} \subset \mathcal{S}(\mathfrak{q})$, we define the transcendence degree of $\mathcal{A}$ as that of the quotient field of $\mathcal{A}$. As is well-known, if $\mathcal{A}$ is commutative, then $\operatorname{trdeg} \mathcal{A} \leqslant b(\mathfrak{q})$. Indeed, if $f_{1}, \ldots, f_{n} \in \mathcal{A}$ are algebraically independent, then $M:=\operatorname{span}\left\{\left(d f_{1}\right)_{\xi}, \ldots,\left(d f_{n}\right)_{\xi}\right\}$ is $n$-dimensional for generic $\xi$. Furthermore, $M$ is an isotropic subspace of $\mathfrak{q}$ with respect to the Kirillov form $\mathcal{K}_{\xi}$. (Recall that $\mathcal{K}_{\xi}(x, y):=\langle\xi,[x, y]\rangle$ and hence $\left.\operatorname{dim}\left(\operatorname{ker} \mathcal{K}_{\xi}\right)=\operatorname{dim} \mathfrak{q}_{\xi}.\right)$

Definition 2. Let $\mathcal{A}$ be a commutative subalgebra of $\mathcal{S}(\mathfrak{q})$. Then $\mathcal{A}$ is said to be of maximal dimension, if $\operatorname{trdeg} \mathcal{A}=b(\mathfrak{q}) ; \mathcal{A}$ is said to be maximal, if it is maximal with respect to inclusion among the commutative subalgebras of $\mathcal{S}(\mathfrak{q})$.

We do not know whether there exist maximal commutative subalgebras that are not of maximal dimension.

Suppose $\mathcal{A}$ is commutative and of maximal dimension. If $\mathcal{A} \subset \mathcal{A}^{\prime}$ and $\mathcal{A}^{\prime}$ is commutative, then each element of $\mathcal{A}^{\prime}$ is algebraic over $\mathcal{A}$. Conversely, if $f \in \mathcal{S}(\mathfrak{q})$ is algebraic over $\mathcal{A}$, then, for generic $\xi \in \mathfrak{q}^{*},(d f)_{\xi}$ belongs to $\operatorname{span}\left\{(d F)_{\xi} \mid F \in \mathcal{A}\right\}$, which is an isotropic subspace with resepect to $\mathcal{K}_{\xi}$. Hence $\{f, F\}(\xi)=0$ for a generic $\xi$ and therefore $\{f, F\} \equiv 0$. Thus, $\mathcal{A}$ is maximal if and only if it is algebraically closed in $\mathcal{S}(\mathfrak{q})$. 
2.2. The argument shift method. Suppose $f \in \mathcal{S}(\mathfrak{q})$ is a polynomial of degree $d$. For any $\xi \in \mathfrak{q}^{*}$, we may consider a shift of $f$ in direction $\xi: f_{a, \xi}(\mu)=f(\mu+a \xi)$, where $a \in \mathbb{k}$. Expanding the right hand side as polynomial in $a$, we obtain the expression $f_{a, \xi}(\mu)=\sum_{j=0}^{d} f_{\xi}^{j}(\mu) a^{j}$. Associated with this shift of argument, we obtain the family of polynomials $f_{\xi}^{j}$, where $j=0,1, \ldots, d-1$. (Since $\operatorname{deg} f_{\xi}^{j}=d-j$, the value $j=d$ is not needed.) We will say that the polynomials $\left\{f_{\xi}^{j}\right\}$ are $\xi$-shifts of $f$. Notice that $f_{\xi}^{0}=f$ and $f_{\xi}^{d-1}$ is a linear form on $\mathfrak{q}^{*}$, i.e., an element of $\mathfrak{q}$. Actually, $f_{\xi}^{d-1}=(d f)_{\xi}$. There is also an obvious symmetry with respect to $\xi$ and $\mu: f_{\xi}^{j}(\mu)=f_{\mu}^{d-j}(\xi)$.

The following observation is due to Mishchenko-Fomenko [7].

Lemma 2.1. Suppose that $h_{1}, \ldots, h_{m} \in \mathcal{Z}(\mathfrak{q})$. Then for any $\xi \in \mathfrak{q}^{*}$, the polynomials

$$
\left\{h_{i, \xi}^{j} \mid i=1, \ldots, m ; \quad j=0,1, \ldots, \operatorname{deg} h_{i}-1\right\}
$$

pairwise commute with respect to the Poisson bracket.

Mishchenko and Fomenko used this procedure for constructing commutative subalgebras of maximal dimension in $\mathcal{S}(\mathfrak{q})$. Given $\xi \in \mathfrak{q}^{*}$ and an arbitrary subset $\mathcal{B} \subset \mathcal{Z}(\mathfrak{q})$, let $\mathcal{F}_{\xi}(\mathcal{B})$ denote the subalgebra of $\mathcal{S}(\mathfrak{q})$ generated by the $\xi$-shifts of all elements of $\mathcal{B}$. Clearly, if $\hat{\mathcal{B}}$ is the subalgebra generated by $\mathcal{B}$, then $\mathcal{F}_{\xi}(\mathcal{B})=\mathcal{F}_{\xi}(\hat{\mathcal{B}})$. By Lemma 2.1, all subalgebras $\mathcal{F}_{\xi}(\mathcal{B})$ are commutative. In particular, subalgebras $\mathcal{F}_{\xi}(\mathcal{Z}(\mathfrak{q}))$ are natural candidates on the rôle of commutative subalgebras of maximal dimension.

For $\mathfrak{g}$ semisimple, it is proved in [7] that there is an open subset $\Omega \subset \mathfrak{g}^{*}$ such that $\mathcal{F}_{\xi}(\mathcal{Z}(\mathfrak{g}))$ is of maximal dimension for any $\xi \in \Omega$. Following [15],[16],[17], the subalgebras $\mathcal{F}_{\xi}(\mathcal{Z}(\mathfrak{g}))$ are said to be Mishchenko-Fomenko subalgebras.

Remark 2.2. The argument shift method is a particular case of a more general construction related to compatible Poisson brackets. Recall that two Poisson brackets on a commutative associative algebra $\mathcal{S}$ are said to be compatible if any linear combination of them is again a Poisson bracket. For $\mathcal{S}=\mathcal{S}(\mathfrak{q})$, we can consider the usual Lie-Poisson bracket $(f, g) \rightarrow\{f, g\}$ and the bracket $(f, g) \rightarrow\{f, g\}_{\xi}$ obtained by "freezing the argument". Here $f, g \in \mathcal{S}(\mathfrak{q})$ and $\xi \in \mathfrak{q}^{*}$ is a fixed element. By definition, $\{f, g\}(\eta):=\left\langle\eta,\left[(d f)_{\eta},(d g)_{\eta}\right]\right\rangle$ and $\{f, g\}_{\xi}(\eta):=\left\langle\xi,\left[(d f)_{\eta},(d g)_{\eta}\right]\right\rangle$. A direct calculation shows that each linear combination $a\{\}+,b\{,\}_{\xi}$ is again a Poisson bracket on $\mathcal{S}(\mathfrak{q})$.

It is easily seen that if $f \in \mathcal{Z}(\mathfrak{q})$ and $f_{b, \xi}(\nu):=f(\nu+b \xi)$, then $f_{b, \xi}$ is a central function with respect to $\{\}+,b\{,\}_{\xi}$. Furthermore, the assignment $f \mapsto f_{b, \xi}$ is a bijection between two centres. It follows that $\mathcal{F}_{\xi}(\mathcal{Z}(\mathfrak{q}))$ is the subalgebra of $\mathcal{S}(\mathfrak{q})$ generated by the centres of all Poisson brackets $\{\}+,b\{,\}_{\xi}, b \in \mathbb{k}$.

2.3. On Bolsinov's criterion and its extension. A general criterion for $\mathcal{F}_{\xi}(\mathcal{Z}(\mathfrak{q}))$ to be of maximal dimension is found by A.V. Bolsinov. Using our terminology, we can express it as follows.

Theorem 2.3 (cf. Bolsinov [1, Theorem 3.1]). Suppose that $\mathfrak{q}$ satisfies the codim-2 property and $\operatorname{trdeg} z(\mathfrak{q})=\operatorname{ind} \mathfrak{q}$. Then the algebra $\mathcal{F}_{\xi}(\mathcal{Z}(\mathfrak{q}))$ is of maximal dimension for any $\xi \in \mathfrak{q}_{\text {reg }}^{*}$. 
Remark 2.4. The above statement requires, however, some explanations. Strictly speaking, Bolsinov does not include the equality $\operatorname{trdeg} z(\mathfrak{q})=$ ind $\mathfrak{q}$ in his Theorem 3.1. But in the paragraph after Definition 2.2 he formulates a condition on the differentials of the functions that are being shifted. This condition is equivalent to this equality.

The algebra $\mathcal{F}_{\xi}(\mathcal{Z}(\mathfrak{q}))$ is of maximal dimension if and only if there is an $\eta \in \mathfrak{q}^{*}$ such that the differentials at $\eta$ of all polynomials in $\mathcal{F}_{\xi}(\mathcal{Z}(\mathfrak{q}))$ span a subspace of dimension $b(\mathfrak{q})$. Clearly, such $\eta$ form an open subset of $\mathfrak{q}^{*}$. For our main result, we need, however, a more precise assertion. Here it is.

Theorem 2.5. Keep the assumptions of Theorem 2.3. Let $P \subset \mathfrak{q}^{*}$ be a plane such that $P \backslash\{0\} \subset \mathfrak{q}_{\text {reg }}^{*}$. Suppose that

$(*) \quad \operatorname{dim} \operatorname{span}\left\{(d f)_{\xi_{0}} \mid f \in \mathcal{Z}(\mathfrak{q})\right\}=$ ind $\mathfrak{q}$ for some $\xi_{0} \in P$.

Then $\operatorname{dim} \operatorname{span}\left\{(d f)_{\eta} \mid f \in \mathcal{F}_{\xi}(\mathcal{Z}(\mathfrak{q}))\right\}=b(\mathfrak{q})$ for any linearly independent $\xi, \eta$ in $P$.

Remark. Condition $(*)$ is open, hence it is satisfied on an open subset of $P$. In many important cases, this condition follows from the other ones (see below). Therefore, there is not much harm in it.

Proof. We apply results of Bolsinov [1] (presented in Appendix A) to the compatible Poisson brackets $\{$,$\} and \{,\}_{\xi}$ on $\mathfrak{q}^{*}$, cf. Remark 2.2. For $\eta \in \mathfrak{q}^{*}$, let $A_{\eta}$ and $B_{\eta}$ be the corresponding skew-symmetric forms on $T_{\eta}^{*}\left(\mathfrak{q}^{*}\right) \cong \mathfrak{q}$. Explicitly, $A_{\eta}(x, y)=$ $\langle\eta,[x, y]\rangle$ and $B_{\eta}(x, y)=\langle\xi,[x, y]\rangle$. It follows that $\left(a A_{\eta}+b B_{\eta}\right)(x, y)=\langle a \eta+b \xi,[x, y]\rangle$ and hence

$$
\operatorname{dim}\left(\operatorname{ker}\left(a A_{\eta}+b B_{\eta}\right)\right)=\operatorname{dim} \mathfrak{q}_{a \eta+b \xi} .
$$

We will identify the 2 -dimensional vector spaces $\mathcal{P}=\operatorname{span}\left\{A_{\eta}, B_{\eta}\right\}$ and $P=$ $\operatorname{span}\{\eta, \xi\} \subset \mathfrak{q}^{*}$ by taking $a A_{\eta}+b B_{\eta}$ to $a \eta+b \xi$.

Set $\mathcal{D}:=\operatorname{span}\left\{(d f)_{\eta} \mid f \in \mathcal{F}_{\xi}(\mathcal{Z}(\mathfrak{q}))\right\}$. Our goal is to prove that $\operatorname{dim} \mathcal{D}=b(\mathfrak{q})$. Recall that $\operatorname{trdeg} \mathcal{S}(\mathfrak{q})^{Q}=$ ind $\mathfrak{q}$. Therefore

$$
\Omega:=\left\{\nu \in \mathfrak{q}^{*} \mid \operatorname{dim} \operatorname{span}\left\{(d f)_{\nu} \mid f \in \mathcal{S}(\mathfrak{q})^{Q}\right\}=\operatorname{ind} \mathfrak{q}\right\}
$$

is a non-empty open subset of $\mathfrak{q}^{*}$. Note that $\Omega$ is conical, i.e., $\nu \in \Omega$ if and only if $t \nu \in \Omega$ for any $t \in \mathbb{k}^{\times}$. By the assumption, $\Omega_{P}:=\Omega \cap P \neq \varnothing$.

From Eq. (2.1), it follows that all nonzero forms in $\mathcal{P}$ have the same rank. Applying Proposition A.4 to $V=\mathfrak{q}$ and $\mathcal{P}=\operatorname{span}\left\{A_{\eta}, B_{\eta}\right\}$ shows that $L=$ $\sum_{(a, b) \neq(0,0)} \operatorname{ker}\left(a A_{\eta}+b B_{\eta}\right)$ is a maximal isotropic subspace of $\mathfrak{q}$ with respect to any nonzero element of $\mathcal{P}$. In particular, $\operatorname{dim} L=b(\mathfrak{q})$. Furthermore, since $\Omega_{P}$ is a non-empty and conical subset of $P \backslash\{0\}$, we deduce from Lemma A.1 that

$$
L=\sum_{(1, b) \in \Omega_{P}} \operatorname{ker}\left(A_{\eta}+b B_{\eta}\right),
$$

where $(1, b)$ is regarded as the point $\eta+b \xi \in P$. Because $\operatorname{dim} \mathcal{D} \leqslant b(\mathfrak{q})$, it suffices to prove that $L \subset \mathcal{D}$. Take any $(1, b) \in \Omega_{P}$ and let $C=\{\}+,b\{,\}_{\xi}$ be the corresponding Poisson bracket on $\mathfrak{q}^{*}$. For any $f \in \mathcal{Z}(\mathfrak{q})$, set $\tilde{f}(\nu):=f(\nu+b \xi)$. Then $(d \tilde{f})_{\eta}=(d f)_{\eta+b \xi}$ and $f \mapsto \tilde{f}$ is a bijection between $\mathcal{Z}(\mathfrak{q})$ and $\mathcal{Z}_{C}(\mathfrak{q})$, the centre of the Poisson algebra $(\mathcal{S}(\mathfrak{q}), C)$. Hence

$$
\mathcal{H}:=\operatorname{span}\left\{(d f)_{\eta+b \xi} \mid f \in \mathcal{Z}(\mathfrak{q})\right\}=\operatorname{span}\left\{(d \tilde{f})_{\eta} \mid f \in \mathcal{Z}_{C}(\mathfrak{q})\right\} \subset \operatorname{ker}\left(A_{\eta}+b B_{\eta}\right) .
$$


Since $\eta+b \xi \in \Omega_{P}$, we have $\operatorname{dim} \mathcal{H}=\operatorname{ind} \mathfrak{q}=\operatorname{dim}\left(\operatorname{ker}\left(A_{\eta}+b B_{\eta}\right)\right)$. Hence $\operatorname{span}\left\{(d \tilde{f})_{\eta} \mid\right.$ $\left.f \in \mathcal{Z}_{C}(\mathfrak{q})\right\}=\operatorname{ker}\left(A_{\eta}+b B_{\eta}\right)$. But each $(d \tilde{f})_{\eta}$ is a linear combination of differentials of elements of $\mathcal{F}_{\xi}$. Therefore $\operatorname{ker}\left(A_{\eta}+b B_{\eta}\right) \subset \mathcal{D}$ whenever $(1, b) \in \Omega_{P}$, and we conclude from Eq. (2.2) that $L \subset \mathcal{D}$. Hence $L=\mathcal{D}$, and we are done.

\section{Maximal commutative subalgebras of $\mathcal{S}(\mathfrak{q})$ and flatness}

First, we prove an auxiliary geometric result. Let $V$ be a finite-dimensional vector space and $P \subset V$ a plane. Suppose $\Omega$ is a conical open subset of $V \backslash\{0\}$ such that $\operatorname{codim}(V \backslash \Omega) \geqslant n \geqslant 2$. Let us say that $P$ is an $\Omega$-plane if $P \backslash\{0\} \subset \Omega$. Given $v \in \Omega$, let $\Omega_{v}$ be the set of all $u$ such that $\mathbb{k} v+\mathbb{k} u \subset V$ is an $\Omega$-plane.

Lemma 3.1. $\Omega_{v}$ is an open subset of $V \backslash\{0\}$ and $\operatorname{codim}\left(V \backslash \Omega_{v}\right) \geqslant n-1$.

Proof. Set $S=V \backslash \Omega$ and consider the projectivisations $\mathbb{P}(S) \subset \mathbb{P}(V)$. Here $\mathbb{P}(S)$ is a projective variety of codimension $\geqslant n$. Write $\bar{v}$ for the image of $v$ in $\mathbb{P}(V)$. Let $C$ be the cone in $\mathbb{P}(V)$ generated by $\bar{v}$ and $\mathbb{P}(S)$. That is, $C$ is the union of all lines through $\bar{v}$ and $y$, where $y$ runs over $\mathbb{P}(S)$. Then $C$ is a projective variety of codimension $\geqslant n-1$, and it follows from the construction that if $\bar{u} \notin C$, then $\mathbb{k} v+\mathbb{k} u$ is an $\Omega$-plane. Thus, $\mathbb{P}\left(\Omega_{v}\right)=\mathbb{P}(V) \backslash C$.

The following is our main result.

Theorem 3.2. Let $\mathfrak{q}$ be an algebraic Lie algebra.

(i) Suppose $\left(\mathfrak{q}\right.$, ad $\left.^{*}\right)$ has the codim-2 property and $\mathcal{Z}(\mathfrak{q})$ contains algebraically independent polynomials $f_{1}, \ldots, f_{l}$, where $l=\operatorname{ind} \mathfrak{q}$, such that $\sum_{i=1}^{l} \operatorname{deg} f_{i}=$ $b(\mathfrak{q})$. Then, for any $\xi \in \mathfrak{q}_{\text {reg }}^{*}, \mathcal{F}_{\xi}(\mathcal{Z}(\mathfrak{q}))=\mathcal{F}_{\xi}\left(f_{1}, \ldots, f_{l}\right)$ is a polynomial algebra of Krull dimension $b(\mathfrak{q})$;

(ii) Furthermore, if $\left(\mathfrak{q}, \mathrm{ad}^{*}\right)$ has the codim-3 property, then $\mathcal{F}_{\xi}(\mathcal{Z}(\mathfrak{q}))$ is a maximal commutative subalgebra of $\mathcal{S}(\mathfrak{q})$.

Proof. To simplify notation, write $\mathcal{F}_{\xi}$ in place of $\mathcal{F}_{\xi}(\mathcal{Z}(\mathfrak{q}))$.

(i) It follows from the assumptions and Theorem 1.2 that $z(\mathfrak{q})=\mathbb{k}\left[f_{1}, \ldots, f_{l}\right]$. Hence $\mathcal{F}_{\xi}=\mathcal{F}_{\xi}\left(f_{1}, \ldots, f_{l}\right)$. By Bolsinov's criterion (Theorem 2.3), $\operatorname{trdeg} \mathcal{F}_{\xi}=b(\mathfrak{q})$ for any $\xi \in \mathfrak{q}_{\text {reg }}^{*}$. Set $\Omega=\left\{\xi \in \mathfrak{q}^{*} \mid\left(d f_{1}\right)_{\xi}, \ldots,\left(d f_{l}\right)_{\xi}\right.$ are linearly independent $\}$. From Theorem 1.2(ii), it follows that $\Omega=\mathfrak{q}_{\text {reg }}^{*}$. Hence codim $\left(\mathfrak{q}^{*} \backslash \Omega\right) \geqslant 2$.

Let $P:=\mathbb{k} \xi+\mathbb{k} \eta \subset \mathfrak{q}^{*}$ be a $\mathfrak{q}_{r e g}^{*}$-plane, i.e., each nonzero element of it belongs to $\mathfrak{q}_{r e g}^{*}$. Since $\Omega=\mathfrak{q}_{\text {reg }}^{*}$, each nonzero point of $P$ satisfies condition $(*)$ of Theorem 2.5. Hence Theorem 2.5 guarantees us that, for any $\eta \in P \backslash \mathbb{k} \xi$, the differentials of the $\xi$-shifts of $f_{1}, \ldots, f_{l}$ at $\eta$ span a subspace of dimension $b(\mathfrak{q})$. Next, in view of the equality $\sum_{i=1}^{l} \operatorname{deg} f_{i}=b(\mathfrak{q})$, the set of all $\xi$-shifts of the $f_{i}$ 's consists of $b(\mathfrak{q})$ elements. It follows that the differentials

$$
\left\{\left(d f_{i, \xi}^{j}\right)_{\eta} \mid i=1, \ldots, l ; \quad j=0,1, \ldots, \operatorname{deg} f_{i}-1\right\}
$$

are linearly independent. This already proves that $\mathcal{F}_{\xi}$ is a polynomial algebra freely generated by the $\left\{f_{i, \xi}^{j}\right\}$ 's . We have also proved the following implication:

if $\mathbb{k} \xi+\mathbb{k} \eta$ is a $\mathfrak{q}_{r e g}^{*}$-plane, then the vectors $\left\{\left(d f_{i, \xi}^{j}\right)_{\eta} \mid i=1, \ldots, l ; j=\right.$ $\left.0,1, \ldots, \operatorname{deg} f_{i}-1\right\}$ are linearly independent. 
(ii) Now $\operatorname{codim}\left(\mathfrak{q}^{*} \backslash \Omega\right) \geqslant 3$. Applying Lemma 3.1 to $V=\mathfrak{q}^{*}, \Omega=\mathfrak{q}_{r e g}^{*}$, and $v=\xi$, we conclude that

$$
\left\{\nu \in \mathfrak{q}_{r e g}^{*} \mid\left(d f_{i, \xi}^{j}\right)_{\nu} \text { are linearly independent }\right\}
$$

is an open subset of $\mathfrak{q}^{*}$ whose complement is of codimension $\geqslant 2$. This means, in turn, that $\left[9\right.$, Theorem 1.1] applies to the polynomial subalgebra $\mathcal{F}_{\xi} \subset \mathcal{S}(\mathfrak{q})$. Therefore, we can conclude that the subalgebra $\mathcal{F}_{\xi}$ is algebraically closed in $\mathcal{S}(\mathfrak{q})$.

Assume that $\mathcal{K}$ is a commutative subalgebra of $\mathcal{S}(\mathfrak{q})$ containing $\mathcal{F}_{\xi}$. Since $\mathcal{F}_{\xi}$ has the maximal possible Krull dimension, $\mathcal{F}_{\xi} \subset \mathcal{K}$ is a an algebraic extension. Because $\mathcal{F}_{\xi}$ is algebraically closed in $\mathcal{S}(\mathfrak{q})$, we obtain $\mathcal{F}_{\xi}=\mathcal{K}$.

Remark 3.3. The codim- 3 property is essential for the maximality of $\mathcal{F}_{\xi}(\mathcal{Z}(\mathfrak{q}))$, see Example 4.1.

It would be interesting to find general conditions that guarantee us that the family of $\xi$-shifts of the free generators of $z(\mathfrak{q})$ form a regular sequence in $\mathcal{S}(\mathfrak{q})$. In the geometric language, this means that we are interested in the property that the natural morphism $\mathfrak{q}^{*} \rightarrow \operatorname{Spec}\left(\mathcal{F}_{\xi}(\mathcal{Z}(\mathfrak{q}))\right) \simeq \mathbb{A}^{b(\mathfrak{q})}$ is flat. It is likely that the assumptions of Theorem 3.2 are sufficient for this. However, we unable to prove this as yet.

Remark 3.4. One can use deformation arguments for proving flatness. We mention an affirmative result for $\mathfrak{s l}_{n}$, which is obtained by combining work of several authors. For an arbitrary reductive $\mathfrak{g}$, there is a general procedure of obtaining new commutative subalgebras of $\mathcal{S}(\mathfrak{g})$ as limits of Mishchenko-Fomenko subalgebras $\mathcal{F}_{\xi}(\mathcal{Z}(\mathfrak{g}))$, where $\xi$ runs inside a fixed Cartan subalgebra of $\mathfrak{g}$, see [15]. In particular, for $\mathfrak{g}=\mathfrak{s l}_{n}$, there is a special limit subalgebra that is the associated graded algebra of the GelfandZetlin subalgebra of $\mathcal{U}\left(\mathfrak{s l}_{n}\right)$, see $[17, \S 6]$. In [8], it is proved that the free generators of the latter form a regular sequence in $\mathcal{S}\left(\mathfrak{s l}_{n}\right)$. This implies that if $\xi \in\left(\mathfrak{s l}_{n}\right)^{*} \simeq \mathfrak{s l}_{n}$ is regular semisimple, then the free generators of $\mathcal{F}_{\xi}\left(Z\left(\mathfrak{s l}_{n}\right)\right)$ form a regular sequence.

\section{Applications}

4.1. Some Lie algebras with codim -3 property. Here we describe several classes of Lie algebras, where Theorem 3.2 applies.

1) If $\mathfrak{g}$ is reductive, then the assumptions of Theorem 3.2 are satisfied. This follows from the classical results of Kostant [4]. Therefore, for any $\xi \in \mathfrak{g}_{r e g}, \mathcal{F}_{\xi}(\mathcal{Z}(\mathfrak{g}))$ is a polynomial algebra, and it is a maximal commutative subalgebra of $\mathcal{S}(\mathfrak{g})$. For the regular semisimple $\xi$, this has already been proved by Tarasov [16].

2) Following [13], recall the definition of a (generalised) Takiff Lie algebra (modelled on $\mathfrak{q}$ ). The infinite-dimensional $\mathbb{k}$-vector space $\mathfrak{q}_{\infty}:=\mathfrak{q} \otimes \mathbb{k}[T]$ has a natural structure of a Lie algebra such that $\left[x \otimes \mathrm{T}^{l}, y \otimes \mathrm{T}^{k}\right]=[x, y] \otimes \mathrm{T}^{l+k}$. Then $\mathfrak{q}_{\geqslant(n+1)}=\bigoplus_{j \geqslant n+1} \mathfrak{q} \otimes \mathrm{T}^{j}$ is an ideal of $\mathfrak{q}_{\infty}$, and $\mathfrak{q}_{\infty} / \mathfrak{q} \geqslant(n+1)$ is a generalised Takiff Lie algebra, denoted $\mathfrak{q}\langle n\rangle$. If $\mathfrak{q}=\mathfrak{g}$ is semisimple, then $\mathfrak{g}\langle n\rangle$ satisfies all the assumptions of Theorem 3.2, see [13]. For $n=1$, one obtains the semi-direct product $\mathfrak{g} \ltimes \mathfrak{g}$. This case was studied by Takiff in 1971 .

3) Let $e \in \mathfrak{s l}_{n}$ be a nilpotent element. Set $\mathfrak{q}=\mathfrak{z}_{\mathfrak{s} l_{n}}(e)$. Then ind $\mathfrak{q}=\operatorname{rk}\left(\mathfrak{s l}_{n}\right)=n-1$ [19] and $\mathcal{S}(\mathfrak{q})^{Q}$ is a polynomial algebra of Krull dimension $n-1$ such that the sum of 
the degrees of free generators equals $b(\mathfrak{q})[9$, Theorem 4.2]. The second author can prove that here $\left(\mathfrak{q}, \mathrm{ad}^{*}\right)$ have codim-3 property. (This will appear elsewhere.) Thus, $\mathfrak{z}_{\mathfrak{s} \mathfrak{l}_{n}}(e)$ satisfies all the assumptions of Theorem 3.2.

4) Let $\mathfrak{q}$ be a $\mathbb{Z}_{2}$-contraction of a simple Lie algebra $\mathfrak{g}$. It is known that $\operatorname{trdeg} z(\mathfrak{q})=\operatorname{ind} \mathfrak{q}[11$, Lemma 2.6$]$ and $\left(\mathfrak{q}, \mathrm{ad}^{*}\right)$ has the codim-2 property [11, Theorem 3.3]. However, the stronger codim -3 property is not always satisfied. Recall the relevant setup.

Let $\mathfrak{g}=\mathfrak{g}_{0} \oplus \mathfrak{g}_{1}$ be a $\mathbb{Z}_{2}$-grading of $\mathfrak{g}$. Then the semi-direct product $\mathfrak{q}=\mathfrak{g}_{0} \ltimes \mathfrak{g}_{1}$ is called a $\mathbb{Z}_{2}$-contraction of $\mathfrak{g}$. Here ind $\mathfrak{q}=$ ind $\mathfrak{g}=\operatorname{rk} \mathfrak{g}$, hence $b(\mathfrak{q})=b(\mathfrak{g})$. For most $\mathbb{Z}_{2}$-gradings, it is proved that $\mathcal{Z}(\mathfrak{q})$ is polynomial and the sum of degrees of free generators equals $b(\mathfrak{g})$, see [11, Sect. 4 \& 5]. It follows that, for such $\mathbb{Z}_{2}$-contractions, the commutative subalgebras $\mathcal{F}_{\xi}(\mathcal{Z}(\mathfrak{q})), \xi \in \mathfrak{q}_{\text {reg }}^{*}$, are polynomial and of maximal dimension. However, these are not always maximal.

Example 4.1. Let $\mathfrak{g}=\mathfrak{g}_{0} \oplus \mathfrak{g}_{1}$ be a $\mathbb{Z}_{2}$-grading such that $\mathfrak{g}_{1}$ contains a Cartan subalgebra of $\mathfrak{g}_{1}$. It is equivalent to that $\operatorname{dim} \mathfrak{g}_{1}=b(\mathfrak{g})$. Then $\mathcal{S}(\mathfrak{q})^{Q}=\mathcal{S}\left(\mathfrak{g}_{1}\right)^{G_{0}} \simeq \mathcal{S}(\mathfrak{g})^{G}$. (This clearly shows that the sum of degrees of free generators of $\mathcal{S}(\mathfrak{q})^{Q}$ equals $b(\mathfrak{g})$.) By the assumption, $\mathfrak{g}_{1}$ contains regular elements of $\mathfrak{g}$ and, hence, of $\mathfrak{q}$. Let $\xi \in \mathfrak{g}_{1}$ be such an element. Then $\mathcal{F}_{\xi}(\mathcal{Z}(\mathfrak{q}))=\mathcal{F}_{\xi}\left(\mathcal{S}\left(\mathfrak{g}_{1}\right)^{G_{0}}\right)$ is a proper subalgebra of $\mathcal{S}\left(\mathfrak{g}_{1}\right)$. Indeed, the family of $\xi$-shifts of the generators contains $b(\mathfrak{g})$ elements, but not all of them are of degree 1 . On the other hand, the subspace $\mathfrak{g}_{1}$ is a commutative Lie subalgebra of $\mathfrak{q}$, hence $\mathcal{S}\left(\mathfrak{g}_{1}\right)$ is a commutative subalgebra of $\mathcal{S}(\mathfrak{q})$. (Actually, it is a maximal commutative subalgebra!) Thus, $\mathcal{F}_{\xi}(\mathcal{Z}(\mathfrak{q}))$ is a commutative subalgebra of $\mathcal{S}(\mathfrak{q})$ of maximal dimension, but not maximal.

Of course, the reason for such a "bad" behaviour is that $\operatorname{codim}\left(\mathfrak{q}^{*} \backslash \mathfrak{q}_{r e g}^{*}\right)=2$. This can also be proved directly using invariant-theoretic properties of the $G_{0}$-module $\mathfrak{g}_{1}$ [5].

Example 4.2. We have verified that the codim-3 property holds for $\mathbb{Z}_{2}$-contractions associated with the following symmetric pairs $\left(\mathfrak{g}, \mathfrak{g}_{0}\right):\left(\mathfrak{s l}_{2 n}, \mathfrak{s p}_{2 n}\right) ;\left(\mathfrak{s l}_{n+1}, \mathfrak{g l}_{n}\right), n \geqslant 2$; $\left(\mathfrak{s o}_{n}, \mathfrak{s o}_{n-1}\right) ;\left(\mathbf{E}_{6}, \mathbf{F}_{4}\right) ;\left(\mathbf{F}_{4}, \mathbf{B}_{4}\right)$. However, the complete list is not known yet. For items 2,3 , and 5 , it is shown in [11] that $Z(\mathfrak{q})$ is polynomial and the sum of degrees of the free generators equals $b(\mathfrak{q})$. Hence Theorem 3.2 applies there.

Remark 4.3. Another criterion for maximality is given by Joseph and Lamprou [2]. They show that if condition (i) of Theorem 0.1 is satisfied and $\xi$ can be included in a so-called adapted pair, then $\mathcal{F}_{\xi}(\mathcal{Z}(\mathfrak{q}))$ is maximal. In [2], adapted pairs are constructed for the so-called truncated parabolic subalgebras of maximal index in $\mathfrak{s l}_{n}$. It is also shown that $z(\mathfrak{q})$ is a polynomial algebra and the equality $\sum \operatorname{deg} f_{i}=b(\mathfrak{q})$ holds. It would be interesting to verify whether the codim-3 property also holds there.

4.2. Semi-direct products and the codim-3 property. Example 4.1 can be put in a more general context. Suppose $G$ is semisimple and $V$ is a finite-dimensional $G$-module. Set $m=\max _{\zeta \in V^{*}} \operatorname{dim} G \cdot \zeta$. Form the semi-direct product $\mathfrak{q}=\mathfrak{g} \ltimes V$.

Proposition 4.4. Suppose that (a) $S(V)^{G}=\mathbb{k}\left[V^{*}\right]^{G}$ is a polynomial algebra and (b) $m=\operatorname{dim} \mathfrak{g}$. Then $\left(\mathfrak{q}, \mathrm{ad}^{*}\right)$ does not satisfy the codim-3 property and the commutative subalgebras $\mathcal{F}_{\xi}(\mathcal{Z}(\mathfrak{q}))$ are not maximal. 
Proof. It follows from assumption (b) and Raïs' formula [12] that ind $\mathfrak{q}=\operatorname{dim} V-$ $\operatorname{dim} \mathfrak{g}$ and therefore $b(\mathfrak{q})=\operatorname{dim} V$. Also, assumption (b) implies that $\mathbb{k}\left[\mathfrak{q}^{*}\right]^{Q}=\mathbb{k}\left[V^{*}\right]^{G}$ $\left[10\right.$, Theorem 6.4]. Thus, $z(\mathfrak{q})=\mathcal{S}(\mathfrak{q})^{Q}=\mathbb{k}\left[\mathfrak{q}^{*}\right]^{Q}$ is a polynomial algebra. Since $G$ has no rational characters, $\mathbb{k}\left(V^{*}\right)^{G}$ is the quotient field of $\mathbb{k}\left[V^{*}\right]^{G}$. Hence $\operatorname{trdeg} \mathbb{k}\left[V^{*}\right]^{G}=$ ind $\mathfrak{q}$. Let $d$ be the sum of degrees of free generators of $\mathbb{k}\left[V^{*}\right]^{G}$. By [3, Korollar 6$]$, $d \leqslant \operatorname{dim} V$. Assume that $\left(\mathfrak{q}, \mathrm{ad}^{*}\right)$ has the codim-3 property. Then $d \geqslant b(\mathfrak{q})=$ $\operatorname{dim} V$ (Theorem 1.2). Hence $d=b(\mathfrak{q})$ and by Theorem 3.2, $\mathcal{F}_{\xi}(\mathcal{Z}(\mathfrak{q}))$ is a maximal commutative subalgebra of $\mathcal{S}(\mathfrak{q})$ for any $\xi \in \mathfrak{q}_{\text {reg }}^{*}$. Since $\mathcal{Z}(\mathfrak{q})$ is a subalgebra of $\mathcal{S}(V), \mathcal{F}_{\xi}(\mathcal{Z}(\mathfrak{q}))$ is a subalgebra of $\mathcal{S}(V)$, too. Furthermore, $\mathcal{F}_{\xi}(\mathcal{Z}(\mathfrak{q}))$ is generated by $\operatorname{dim} V$ elements, and not all of them are of degree 1 . Thus, $\mathcal{F}_{\xi}(\mathcal{Z}(\mathfrak{q}))$ is a proper subalgebra of $\mathcal{S}(V)$, and the latter is a (maximal) commutative subalgebra of $\mathcal{S}(\mathfrak{q})$. This contradiction shows that the codim-3 property cannot be satisfied for $\left(\mathfrak{q}, \mathrm{ad}^{*}\right)$. The above argument also proves the second assertion.

Remark 4.5. Set $V_{\text {sing }}^{*}=\left\{\nu \in V^{*} \mid \operatorname{dim} G \cdot \nu<m\right\}$. (This closed subset plays an important rôle in theory developed in [3].) It is easily seen that if $m=\operatorname{dim} G$ and $\operatorname{codim} V_{\text {sing }}^{*} \geqslant n$, then codim $\mathfrak{q}^{*} \backslash \mathfrak{q}_{r e g}^{*} \geqslant n$. Hence, under the assumptions of Proposition 4.4, we have codim $V_{\text {sing }}^{*} \leqslant 2$, and according to [3, Korollar 2], $\operatorname{codim} V_{\text {sing }}^{*}=2$ if and only if $d=b(\mathfrak{q})$.

\section{Appendix A. Some results on skew-symmetric bilinear forms}

Here we present some general facts concerning skew-symmetric bilinear forms that are needed for the proof of Theorem 2.5. All these results are extracted from [1], but we present them in a more systematic form.

Let $\mathcal{P}$ be a two-dimensional linear space of (possibly degenerate) skew-symmetric bilinear forms on a finite-dimensional vector space $V$. Set $m=\max _{A \in \mathcal{P}} \operatorname{rk} A$, and let $\mathcal{P}_{\text {reg }} \subset \mathcal{P}$ be the set of all forms of rank $m$. For each $A \in \mathcal{P}$, let ker $A \subset V$ be the kernel of $A$. Our main object of interest is the subspace $L:=\sum_{A \in \mathcal{P}_{\text {reg }}} \operatorname{ker} A$.

Lemma A.1. For any nonempty open subset $\Omega \subset \mathcal{P}_{\text {reg }}$, we have $\sum_{A \in \Omega} \operatorname{ker} A=L$.

Proof. Set $r=\operatorname{dim} V-m$ and $M=\sum_{A \in \Omega} \operatorname{ker} A \subset L$. Take any $C \in \mathcal{P}_{\text {reg }} \backslash \Omega$. Then ker $C$ is a point of the Grassmannian $\operatorname{Gr}_{r}(V)$. Because $\mathcal{P}$ is irreducible, $\bar{\Omega}=\mathcal{P}$ and there is a curve $\varkappa: \mathbb{k}^{\times} \rightarrow \Omega$ such that $\lim _{t \rightarrow 0} \varkappa(t)=C$. Hence

$$
\lim _{t \rightarrow 0}(\operatorname{ker} \varkappa(t))=\operatorname{ker} C,
$$

where the last limit is taken in $\operatorname{Gr}_{r}(V)$. Since $\operatorname{ker} \varkappa(t) \in \operatorname{Gr}_{r}(M)$ for $t \neq 0$ and $\operatorname{Gr}_{r}(M)$ is closed in $\operatorname{Gr}_{r}(V)$, we obtain $\operatorname{ker} C \subset M$. Thus, $M=L$.

For $A \in \mathcal{P}$, let $\hat{A}$ denote the corresponding linear map from $V$ to $V^{*}$. Then $\operatorname{ker} A=$ ker $\hat{A}$.

Lemma A.2. For all $A, B \in \mathcal{P} \backslash\{0\}$, we have $\hat{A}(L)=\hat{B}(L)$.

Proof. Clearly, we may assume that $A$ and $B$ are linearly independent. By virtue of Lemma A.1, $L$ is spanned by some $L_{a, b}:=\operatorname{ker}(a A+b B)$ with $a b \neq 0$. Since $(a \hat{A}+b \hat{B})\left(L_{a, b}\right)=0$, we obtain $(a \hat{A})\left(L_{a, b}\right)=(b \hat{B})\left(L_{a, b}\right)$ and hence $\hat{A}\left(L_{a, b}\right)=\hat{B}\left(L_{a, b}\right)$. The result follows. 
For $A \in \mathcal{P} \backslash\{0\}$, let $\widetilde{L} \subset V$ denote the annihilator of $\hat{A}(L) \subset V^{*}$. By Lemma A.2, $\widetilde{L}$ does not depend on the choice of $A$. Note also that $\widetilde{L}=\{v \in V \mid A(v, L)=0\}$. Since $\operatorname{ker} A \subset \widetilde{L}$ for each nonzero $A, L$ is a subspace of $\widetilde{L}$.

Lemma A.3. Suppose that $B \in \mathcal{P}$ and $A \in \mathcal{P}_{\text {reg. }}$. Then

(i) $\hat{B}(\widetilde{L}) \subset \hat{A}(\widetilde{L})$;

(ii) Associated with $A$ and $B$, there is a natural linear operator $\Phi_{A, B}=\Phi$ : $\widetilde{L} / L \rightarrow \widetilde{L} / L$.

Proof. (i) Let $M_{A}$ and $M_{B}$ be the the annihilators of $\hat{A}(\widetilde{L})$ and $\hat{B}(\widetilde{L})$, respectively. Since $M_{A}=\operatorname{ker} A+L=L$ and $M_{B}=\operatorname{ker} B+L$, we obtain $M_{A} \subset M_{B}$.

(ii) Take any $v \in \widetilde{L}$. Since $\hat{B}(\widetilde{L}) \subset \hat{A}(\widetilde{L})$, where is $w \in \widetilde{L}$ such that $\hat{A}(w)=\hat{B}(v)$. Letting $\Phi(v+L):=w+L$, we have to check that there is no ambiguity in this. To this end, assume that $\hat{A}\left(w^{\prime}\right) \in \hat{B}(v+L)=\hat{A}(w)+\hat{B}(L)$. Since $\hat{B}(L)=\hat{A}(L)$, we obtain $\hat{A}\left(w^{\prime}-w\right) \in \hat{A}(L)$. Hence $w-w^{\prime} \in L+\operatorname{ker} A=L$. Thus, given $\bar{v}=v+L \in \widetilde{L} / L$, there is a unique $\bar{w}=w+L \in \widetilde{L} / L$ such that $\hat{B}(\bar{v})=\hat{A}(\bar{w})$. The claim follows.

Proposition A.4. If $\mathcal{P}_{\text {reg }}=\mathcal{P} \backslash\{0\}$, then $L=\widetilde{L}$; in other words, $L$ is a maximal isotropic subspace of $V$ with respect to any nonzero $A \in \mathcal{P}$.

Proof. Take linearly independent $A$ and $B$, as in Lemma A.3. We use the operator $\Phi: \widetilde{L} / L \rightarrow \widetilde{L} / L$ introduced in Lemma A.3(ii). Since $\mathbb{k}$ is algebraically closed, $\widetilde{L} / L=$ $\{0\}$ if and only if all eigenvectors of $\Phi$ are zero. Assume that $v+L \in \widetilde{L} / L$ is a $\lambda$ eigenvector of $\Phi$. Then expanding the definition of $\Phi$ yields $(\hat{B}-\lambda \hat{A}) v \in \hat{A}(L)$. Since $\hat{A}(L)=(\hat{B}-\lambda \hat{A})(L)$ by Lemma A.2, we get $(\hat{B}-\lambda \hat{A})(v) \in(\hat{B}-\lambda \hat{A})(L)$ and, hence, $v \in L+\operatorname{ker}(B-\lambda A)$. If $v \notin L$, then $\operatorname{ker}(B-\lambda A) \not \subset L$ and therefore $(B-\lambda A) \notin \mathcal{P}_{\text {reg }}$. A contradiction!

\section{References}

[1] A.V. Bolsinov, Commutative families of functions related to consistent Poisson brackets, Acta Appl. Math. 24 (1991), no. 3, 253-274.

[2] A. Joseph and P. Lamprou, Maximal Poisson commutative subalgebras for truncated parabolic subalgebras of maximal index in $\mathfrak{s l}_{n}$, Transformation Groups, 12 (2007), 549-571.

[3] F. Knop, Über die Glattheit von Quotientenabbildungen, Manuscr. Math. 56 (1986), 419-427.

[4] B. Kostant, Lie group representations in polynomial rings, Amer. J. Math. 85(1963), 327-404.

[5] B. Kostant and S. Rallis, Orbits and representations associated with symmetric spaces, Amer. J. Math. 93 (1971), 753-809.

[6] B. Kostant and N. Wallach, Gelfand-Zeitlin theory from the perspective of classical mechanics, I. in: "Studies in Lie Theory", Basel: Birkhäuser, Progress in Math. 243 (2006), 319-364.

[7] А.С. Мищенко, А.Т. Фоменко, Уравнения Эйлера на конечномерных группах Ли, Изв. АН СССР. Сер. Матем. 42, № 2 (1978), 396-415 (Russian). English translation: A.S. Mishchenko and A.T. Fomenko. Euler equation on finite-dimensional Lie groups, Math. USSR-Izv. 12 (1978), 371-389.

[8] S. Ovsienko, Strongly nilpotent matrices and Gelfand-Zetlin modules, Linear Algebra Appl. 365 (2003), 349-367.

[9] D. Panyushev, A. Premet, and O. Yakimova, On symmetric invariants of centralisers in reductive Lie algebras, J. Algebra 313 (2007), no. 1, 343-391.

[10] D. Panyushev, Semi-direct products of Lie algebras and their invariants, Publ. RIMS, 43 (2007), 1199-1257. 
[11] Math. 213 (2007), no. 1, 380-404.

[12] M. Raïs, L'indice des produits semi-directs $E \times_{\rho} \mathfrak{g}$, C.R. Acad. Sc. Paris, Ser. A. 287 (1978), 195-197.

[13] M. Raïs and P. Tauvel, Indice et polynômes invariants pour certaines algèbres de Lie, J. Reine Angew. Math. 425 (1992), 123-140.

[14] Л.Г. Рыбников. Метод сдвига инвариантов и модель Годена. Функи. анализ и его прилож., 40, № 3 (2006), 30-43 (Russian). English translation: L.G. RYBNikov. The argument shift method and the Gaudin Model, Funct. Anal. Appl. 40 (2006), 188-199.

[15] В.В. Шувалов. О пределах подалгебр Мищенко-Фоменко в алгебрах Пуассона полупростых алгебр Ли, Функи. анализ и его прилож., 36, № 4 (2002), 55-64 (Russian). English translation: V.V. Shuvalov. On limits of Mishchenko-Fomenko subalgebras in Poisson algebras of semisimple Lie algebras, Funct. Anal. Appl. 36 (2002), 298-305.

[16] А.А. Тарасов. Максимальность некоторых коммутативных подалгебр в алгебрах Пуассона полупростых алгебр Ли, УМH 57, № 5 (2002), 165-166 (Russian). English translation: A.A. TARASOV. The maximality of certain commutative subalgebras in Poisson algebras of a semisimple Lie algebra, Russian Math. Surveys 57 (2002), no. 5, 1013-1014.

[17] Э.Б. Винберг. О некоторых коммутативных подалгебрах универсальной обертывающей алгебры, Изв. АН СССР. Сер. Матем. 54, № 1 (1990), 3-25 (Russian). English translation: E.B. VINBERG. On certain commutative subalgebras of a universal enveloping algebra, Math. USSR-Izv. 36 (1991), 1-22.

[18] Э.Б. Винберг, А.Л. Онищик. Семинар по группам Ли и алгебраическим группам. Москва: "Наука" 1988 (Russian). English translation: A.L. ONishChiK and E.B. VinBERG. "Lie groups and algebraic groups", Berlin: Springer, 1990.

[19] О.С. Якимова. Индекс централизаторов элементов в классических алгебрах Ли, Функи. анализ и его прилож., 40, № 1 (2006), 52-64 (Russian). English translation: O.S. Yakimova. The index of centralisers of elements in classical Lie algebras, Funct. Anal. Appl., 40 (2006), 42-51.

(D.P.) Independent University of Moscow, Bol'shoi Vlasevskil Per. 11, 119002 Moscow, Russia

E-mail address: panyush@mccme.ru

(O.Y.) Universität Zu KÖln, Mathematisches Institut, Weyertal 86-90, D-50931 Köln, Deutschland

E-mail address: oyakimov@math.uni-koeln.de 